\title{
次
}

Ángela Andrea Caviedes Conde*

Luis Ramón Yanguas Morte**

\section{UN MODELO PARA EL ESTUDIO DE LAS VARIABLES ECONÓMICAS QUE INFLUYEN EN LA AYUDA OFICIAL AL DESARROLLO}

En este artículo, una vez descritas la evolución y características de la Ayuda Oficial al Desarrollo, se presenta un modelo econométrico descriptivo de determinadas variables sobre dicha ayuda. Este modelo está basado en las líneas generales descritas en un artículo anterior (BICE 3098) empleando las mismas fuentes estadísticas. Destacamos entre las variables el nivel de endeudamiento externo, la apertura comercial, el PIB per cápita y el acceso a capitales privados.

Palabras clave: Ayuda Oficial al Desarrollo, objetivos del desarrollo, objetivos del milenio.

Clasificación JEL: F10, F63, O10.

\section{Introducción}

Se han proseguido nuestras investigaciones para completar la anterior publicada en el BICE n. .3093 , ampliándolas con la presentación de un mayor número de variables económicas con incidencia en el desarrollo. En este trabajo presentamos un estudio econométrico que complementa el anterior.

\section{El carácter compensatorio de la Ayuda Oficial al Desarrollo}

A pesar de los inconvenientes puestos de manifiesto en el análisis de las tendencias

\footnotetext{
* Profesora de Marketing y Negocios Internacionales. Universidad Internacional de La Rioja. Doctora por la Universidad de Alcalá.

** Director de Finanzas. BCD TRAVEL, Atlanta, GA, Estados Unidos. Doctor por la Universidad de Alcalá. CPA, CMA.

Versión de diciembre de 2017.
}

de la Ayuda Oficial al Desarrollo (AOD), tales como la tendencia de reducción de la Ayuda de Programa, Proyecto y Asistencia Técnica bilateral (que supone entradas reales y previsibles y no condonaciones o asistencias puntuales) o el todavía elevado sesgo de concentración en torno a receptores como Irak, Afganistán y Etiopía, etcétera, podemos decir que dentro del conjunto de instrumentos tradicionales de financiación al desarrollo, la Ayuda Oficial al Desarrollo tiene un papel principal en la consecución de los Objetivos de Desarrollo Sostenible de los países en desarrollo y con menores niveles de renta, especialmente los países menos adelantados, descritas anteriormente en el análisis tanto del marco teórico como de las tendencias de la AOD.

Dichas ventajas se pueden resumir en: i) altas tasas de crecimiento con menores $\square$ 
niveles de volatilidad que los capitales privados; ii) menor grado de condicionalidad que los capitales privados, permitiendo al país el empleo en aquellos proyectos más prioritarios; iii) alto grado de donaciones (50 por 100 para los países menos adelantados), permitiendo que el acceso a dichos fondos no suponga un empeoramiento de la posición deudora internacional del país receptor; iv) menor concentración de países receptores que los capitales privados y, finalmente, v) la aportación de fondos para el sostenimiento de los bienes públicos globales.

La situación actual se caracteriza por la escasez de recursos, debida principalmente al impacto del actual contexto económico global sobre los países (donantes bilaterales) y a la reducción de los recursos disponibles de los organismos multilaterales (exhaustos ante los fondos aplicados a las medidas anticrisis en los últimos años, los compromisos existentes de condonación de deuda, la asistencia humanitaria y la ayuda de emergencia). Esta situación supone un incremento de la dificultad de los países para acceder a los recursos financieros necesarios para el desarrollo, especialmente para aquellos países que no figuren dentro de las listas del Comité de Ayuda al Desarrollo.

A lo largo del siglo xxI la AOD ha mostrado un crecimiento sostenido con un componente de donaciones superior al 90 por 100 y ha estado principalmente orientada a los países de renta baja y medio baja, especialmente a África, resultando interesante entender, por un lado, el impacto de los compromisos internacionales de asistencia oficial para el desarrollo de los Objetivos de Desarrollo del Milenio, el Programa de Acción de países menos adelantados de Bruselas 2001, el Programa de Acción de desarrollo sostenible de pequeños Estados insulares en desarrollo de Mauricio 2005, el Programa de Eficacia de la Ayuda de Accra 2008 y el Programa de Acción de países menos adelantados de Estambul 2011, entre otros; y por otro lado, iniciativas tales como el Plan Estratégico África 2009-2012 de la Unión Africana, el Programa de Acción en favor de países menos adelantados 2011-2020, siendo por tanto interesante y relevante entender qué factores descriptivos de dichos países han tenido un mayor impacto sobre los flujos de entrada de AOD para entender mejor qué opciones tienen ante sí los países en desarrollo, los países menos adelantados, los pequeños Estados insulares en desarrollo y los países en desarrollo sin litoral para seguir incrementando dichos flujos de entrada.

Por tanto, el objetivo de este análisis es entender cuáles son los factores determinantes de la entrada de flujos de Ayuda Oficial al Desarrollo en el país, para lo cual, y en base a lo expuesto hasta ahora en este documento, la hipótesis de partida fijada es que la AOD tiene un efecto compensatorio, siendo atraída allí donde existe menor acceso a financiación privada e internacional.

Las limitaciones principales del modelo se deben principalmente a la propia extensión de este trabajo, a la falta de datos suficientes de determinadas variables relevantes dentro del marco temporal y para el conjunto de países seleccionado y, finalmente, a la necesidad de evitar un elevado grado de complejidad que impida interpretar los resultados obtenidos.

En este sentido, se subraya que el trabajo adolece, por un lado, de ciertas variables independientes más específicas, como, por ejemplo, un análisis de datos concretos de inversión entre economías receptoras y emisoras en relación al comercio internacional y la Ayuda $\triangleright$ 
Oficial al Desarrollo, la inclusión en el modelo del impacto de los conflictos armados o los desastres naturales, y por otro lado, de un mayor nivel de detalle, como puede ser la apertura de datos a nivel sectorial o las tipologías de ayuda, entre otras cuestiones.

\subsection{Elección de las variables independientes relacionadas}

Las variables independientes seleccionadas en los modelos empleados en el contraste de la hipótesis definida están descritas en detalle en el Anexo 1. Para la elección de dichas variables independientes como factores determinantes de los flujos de entrada de Ayuda Oficial al Desarrollo, se han agrupado en cinco categorías: i) las condiciones macroeconómicas y riesgos, ii) la apertura comercial, iii) el desarrollo financiero, iv) la demografía y v) el nivel de renta.

i) Las condiciones macroeconómicas y riesgos en base a lo ya indicado en el artículo sobre impacto de las inversiones privadas en el desarrollo de los países en desarrollo, en el número 3093 de 2017 de esta revista, en la que se señala que se pueden determinar a priori el volumen de entradas de AOD, especialmente la orientada a programas y proyectos.

En el presente análisis se han elegido como índices representativos de las condiciones macroeconómicas de un país el tipo de cambio y la inflación (porcentaje) que se espera, que el tipo de cambio tenga un impacto positivo y significativo y que la inflación, en cambio, muestre una influencia negativa sobre las entradas de AOD.

ii) La apertura comercial de manera similar a lo expuesto en el mencionado Boletín de ICE (número 3093; 2017). La apertura comercial puede ser considerada como determinante de los flujos de entrada de Ayuda Oficial al Desarrollo, especialmente bilateral. En el presente análisis se tendrá en cuenta el volumen de exportaciones en porcentaje sobre el PIB como variable independiente del modelo, ya que ha mostrado una significación importante en estudios realizados por diversos autores, como Bandyopadhyay y Wall (2007), en base a los cuales se espera que todos tengan una relación positiva con las entradas de AOD.

iii) El desarrollo del sistema financiero en los países receptores tiene implicaciones significativas en relación con el volumen de entradas netas de AOD. Los compromisos de los organismos oficiales y de los países donantes están orientados a ofrecer más ayuda y más eficaz a aquellos países con menores recursos y con menor acceso a otras fuentes de financiación, de manera que a priori un país con un mayor desarrollo financiero tendrá mayor capacidad de endeudamiento y de acceso a crédito privado y público, y debe, por tanto, recibir menor volumen de AOD que otro con las mismas necesidades y menor nivel de desarrollo financiero, tal y como se establece en diversos informes internacionales.

En el presente análisis se han elegido los índices representativos del desarrollo financiero, el volumen de entradas de capitales privados en porcentaje sobre el PIB, el volumen de entradas de deuda exterior, el volumen de crédito al sector privado, el volumen de crédito al sector bancario y el volumen de masa monetaria, ya que han mostrado una significación importante en estudios realizados por diversos autores, como Marcuello Servos, Clemente López y Filgueira Chiossoni (2010), en base a los cuales se espera que todos muestren $\triangleright$ 
una relación negativa con las entradas de Ayuda Oficial al Desarrollo.

iv) El tamaño de mercado y la demografía pueden influir en las entradas de AOD. En el presente análisis se ha elegido como índice representativo de la demografía la población total, ya que ha mostrado una significación importante en estudios realizados por diversos autores, como el análisis de Koch et al. (2008), en base a los cuales no está suficientemente claro si existirá una relación positiva con las entradas de AOD.

v) El nivel de renta en términos generales tiene una influencia significativa en la atracción de entradas de AOD. Tanto los países donantes como los organismos multilaterales tienen una motivación por entregar ayuda a los países más necesitados, por ello este indicador refleja el bienestar de la población de un país, de manera que las economías con menores niveles de renta recibirán a priori mayores volúmenes de entrada de AOD.

Dentro del conjunto de factores representativos de esta categoría se recogen índices como el PIB total y el PIB per cápita, u otros indicadores de acceso a servicios básicos como sanidad, educación, alimentación, saneamientos y electricidad, entre otros.

En el presente análisis se ha elegido como índice representativo del nivel de renta el PIB per cápita, ya que ha mostrado una significación importante en estudios realizados por diversos autores, como el análisis de Koch et al. (2008), en base a los cuales se espera que todos tengan una relación negativa con las entradas de AOD.

\subsection{Datos y especificaciones del modelo}

Recordando nuevamente que la hipótesis de partida fijada es que la AOD tiene un efecto compensatorio, siendo atraída allí donde existe menor acceso a financiación privada e internacional, cabe indicar que en la construcción del modelo inicial, como puede observarse en su ecuación, se han empleado diez variables independientes seleccionadas de las cinco categorías de factores con capacidad de influencia en las entradas de AOD antes comentadas y cuya definición detallada puede verse en el Anexo 1.

Todas las variables independientes y la variable dependiente han sido transformadas en logaritmos para acercar su distribución a una normal.

La ecuación del modelo construido es la siguiente:

$$
\begin{aligned}
& A O D=\beta O+\beta 1 T C O F+\beta 2 I P C+\beta 3 \text { EXPOR + } \\
& +\beta 4 P I B P C+\beta 5 P O B+\beta 6 D E X T+\beta 7 B M+ \\
& +\beta 8 K P R I V+\beta 9 D C B A N+\beta 10 D C P R I+\mu i t
\end{aligned}
$$

Siendo:

$\beta O=$ coeficiente de la intersección en el origen.

$\beta n(1-10)=$ coeficiente que acompaña $a$ cada variable independiente.

$\mu$ it = término de error en el que se considera la heterogeneidad transversal no observada, el término de error clásico y los efectos temporales inobservables.

El análisis se ha realizado sobre una muestra recopilada de datos de diversas fuentes, principalmente la base de datos World Development Indicators, y, entre otras, el Banco Mundial, el Fondo Monetario Internacional, el Comité de Asistencia al Desarrollo y las Naciones Unidas. Los datos tienen un periodo de seis años, entre 2005 y 2010 , y un total $D$ 
de 61 países $^{1}$. La tabla de resultados con los coeficientes fijos por variables, así como las correlaciones, se pueden consultar en el Anexo 3.

En la construcción del modelo para su análisis por submuestras, tal y como puede observarse en su ecuación, se han empleado cuatro variables independientes seleccionadas de las cinco categorías de factores con capacidad de influencia en las entradas de AOD antes comentadas y cuya definición detallada puede verse en el Anexo 1. Tanto la variable dependiente como tres de las variables independientes (L-KPRIV, «Porcentaje de entradas de capitales privados sobre el PIB»; L-DEXT, «volumen de entrada de deuda exterior», y L-PIBpc, «PIB per cápita») han sido transformadas en logaritmos para acercar su distribución a una normal.

La ecuación del modelo de submuestras construido es la siguiente:

$$
\begin{gathered}
A O D=\beta O+\beta 1 E X P O R+\beta 2 P I B p c+ \\
+\beta 3 D E X T+\beta 4 K P R I V+\mu i t
\end{gathered}
$$

Siendo:

$\beta 0=$ coeficiente de la intersección en el origen.

$\beta n(1-4)=$ coeficiente que acompaña a cada variable independiente.

$\mu$ it $=$ término de error en el que se considera la heterogeneidad transversal no observada, el

\footnotetext{
1 Los países recogidos en la muestra son: Zambia (ZMB), Yemen (YEM), Vietnam (VNM), Venezuela (VEN), San Vicente y las Granadinas (VCT), Uruguay (URY), Ucrania (UKR), Uganda (UGA), Tanzania (TZA), Turquía (TUR), Túnez (TUN), Tailandia (THA), El Salvador (SLV), Islas Salomón (SLB), Sudán (SDN), Papúa Nueva Guinea (PNG), Filipinas $(\mathrm{PHL})$, Perú (PER), Panamá (PAN), Pakistán (PAK), Nicaragua (NIC), Malasia (MYS), Mauricio (MUS), Mozambique (MOZ), Mongolia (MNG), Macedonia (MKD), México (MEX), Moldavia (MDA), Marruecos (MAR), Lesoto (LSO), Sri Lanka (LKA), Santa Lucía (LCA), St. Kitts y Nevis (KNA), Kenia (KEN), Kazajstán (KAZ), Jordania (JOR), India (IND), Indonesia (IDN), Honduras (HND), Guatemala (GTM), Granada (GRD), Ghana (GHA), Georgia (GEO), Egipto (EGY), Ecuador (ECU), República Dominicana (DOM), Dominica (DMA), Costa Rica (CRI), Cabo Verde $(\mathrm{CPV})$, Colombia (COL), Camerún (CMR), China (CHN), Brasil (BRA), Bolivia (BOL), Bielorrusia (BLR), Bangladesh (BGD), Azerbaiyán (AZE), Armenia (ARM), Argentina (ARG), Albania (ALB), Angola (AGO).
}

término de error clásico y los efectos temporales inobservables.

El análisis se ha realizado sobre una muestra de un total de 86 países que, como puede observarse en el Anexo 2, aparecen agrupados tanto en una muestra global como en un total de doce submuestras que permiten su análisis tanto por tipo de economía como por tipo de región.

\subsection{Resultados empíricos}

El resultado obtenido del análisis del modelo inicial confirma la hipótesis de partida fijada, en la que la $A O D$ tiene un efecto compensatorio, siendo atraída allí donde existe menor acceso a financiación privada e internacional, al probar con un nivel de ajuste medio la influencia de determinadas variables sobre las entradas de AOD, como ponen de manifiesto las correlaciones observadas, especialmente en el caso del nivel de población, el PIB per cápita, el volumen de endeudamiento de la economía, el tipo de cambio oficial y el volumen de exportaciones representativos de la demografía, el nivel de renta y el desarrollo financiero, respectivamente, y en menor medida las observadas para las condiciones macroeconómicas y riesgos y la apertura comercial en último lugar.

Respecto a la muestra de 61 países, principalmente en desarrollo, de renta media y de prácticamente la totalidad de regiones, así como la complejidad del modelo con diez variables independientes, no impide reflejar el resultado del mayor grado de influencia y significación que determinadas variables muestran dentro del modelo.

De esta manera, podemos inferir que las decisiones de asignación de Ayuda Oficial al Desarrollo de Gobiernos, organizaciones $\triangleright$ 
internacionales, organizaciones no gubernamentales, empresas multinacionales, fundaciones y demás miembros de la sociedad civil se ven influenciadas especialmente por: 1) el volumen de PIB per cápita, reflejo de las necesidades de recursos económicos y nivel de renta del país de destino; 2) el volumen de población, que determina el factor demográfico y la escala de necesidades el país receptor; 3) el volumen de exportaciones que determina hasta cierto punto la apertura comercial, el compromiso y la política de desarrollo del país receptor, así como permite mayores retornos por eficiencias y menores riesgos; y finalmente, en menor medida, 4) la inflación, el nivel de endeudamiento y el volumen de capitales privados que determinan la salud económica, estabilidad macro y menor riesgo, así como nivel de desarrollo del sistema financiero y volumen de recursos financieros internacionales disponibles.

Del análisis de la muestra global del modelo de submuestras podemos concluir que confirma la hipótesis nula al probar, con un nivel de ajuste muy alto, la influencia de todas las variables sobre las entradas de AOD, como ponen de manifiesto las altas correlaciones observadas en el Anexo 3.

Dentro de la muestra de 86 países, por número predominan los países en desarrollo, y las regiones de América Latina y Caribe y África Subsahariana, si bien por volumen de entradas de AOD la mayor influencia es de África Subsahariana, en torno a un tercio del total. La presencia de los países menos adelantados tiene una influencia muy importante motivada por el compromiso de incrementos de ayuda durante el periodo derivados de los Objetivos de Desarrollo del Milenio y de los Acuerdos de Gleneagles.

El grado de influencia y significación dentro del modelo muestra cómo la Ayuda Oficial al
Desarrollo se ve atraída principalmente por: 1) el menor volumen de PIB per cápita, como reflejo del menor nivel de desarrollo, en cumplimiento de los compromisos internacionales de incremento de la ayuda a los países más necesitados, como en los países menos adelantados y en la región de África Subsahariana durante el periodo; 2) el mayor volumen de endeudamiento externo por reflejar la situación de los países pobres muy endeudados receptores de las diferentes iniciativas de condonación de deuda; 3 ) el menor volumen de exportaciones reflejo de una menor apertura comercial, bien por la falta de políticas comerciales, recursos, tejido industrial o infraestructuras; $y$, finalmente, 4) el menor acceso a capitales privados que refleja unos sistemas y mercados financieros locales insuficientemente desarrollados, o la falta de atracción de las inversiones externas por no disponer de un tamaño suficiente de mercado, recursos u otros incentivos a la inversión. Este modelo carece de la suficiente significación y representatividad por años y para los niveles de renta baja (Cuadro 1).

En el análisis de la muestra de países en desarrollo de 85 países, con representatividad de todas las regiones de la muestra global, el nivel de ajuste del modelo es muy alto. El resultado confirma la hipótesis nuevamente, y en comparación con la submuestra global la correlación de las variables con las entradas de Ayuda Oficial al Desarrollo se mantiene.

El grado de influencia y significación dentro del modelo muestra cómo en todos los niveles de renta la asignación de la AOD está influenciada principalmente por el mayor endeudamiento externo, el menor PIB per cápita, el menor volumen de exportaciones y el menor acceso a capitales privados.

En relación al análisis de la muestra de países menos adelantados de 16 países, con $\triangleright$ 
UN MODELO PARA EL ESTUDIO DE LAS VARIABLES ECONÓMICAS QUE INFLUYEN...

CUADRO 1

\begin{tabular}{|c|c|c|c|c|c|c|c|c|}
\hline \multirow{2}{*}{ 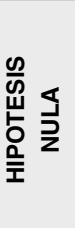 } & \multirow{2}{*}{ MUESTRA } & \multicolumn{3}{|c|}{$\begin{array}{l}\text { NIVEL DE RENTA } \\
\text { DEL PAÍS }\end{array}$} & \multirow{2}{*}{$\begin{array}{l}\text { INFLUENCIA DE DETERMINADAS } \\
\text { VARIABLES SOBRE LA AYUDA OFICIAL } \\
\text { DE DESARROLLO SEGÚN EL NIVEL DE } \\
\text { IMPORTANCIA }\end{array}$} & \multicolumn{3}{|c|}{$\begin{array}{c}\text { NIVEL } \\
\text { DE AJUSTE } \\
\text { DEL MODELO }\end{array}$} \\
\hline & & $\stackrel{5}{\rightleftarrows}$ & 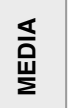 & 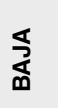 & & 은 & 음 & 옳 \\
\hline \multirow{5}{*}{ 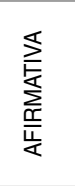 } & \multirow{5}{*}{61 países } & & & & 1. Volumen del PIB per cápita & & & \\
\hline & & & & & 2. Factor demográfico & & & \\
\hline & & & & & 3. Nivel de apertura comercial & & & \\
\hline & & & & & 4. Nivel de acceso a capitales privados & & & \\
\hline & & & & & 5. Nivel de endeudamiento externo & & & \\
\hline \multirow{4}{*}{ 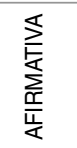 } & \multirow{4}{*}{$\begin{array}{l}86 \text { países en desarrollo. Predominan países de } \\
\text { América Latina, Caribe y África Subsahariana }\end{array}$} & & & & 1. Volumen del PIB per cápita & & & \\
\hline & & & & & 2. Nivel de endeudamiento externo & & & \\
\hline & & & & & 3. Nivel de apertura comercial & & & \\
\hline & & & & & 4. Nivel de acceso a capitales privados & & & \\
\hline \multirow{4}{*}{ 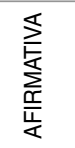 } & \multirow{4}{*}{$\begin{array}{l}85 \text { países, representación de todas las regiones } \\
\text { de la muestra global }\end{array}$} & & & & 1. Nivel de endeudamiento externo & & & \\
\hline & & & & & 2. Nivel del PIB per cápita & & & \\
\hline & & & & & 3. Nivel de apertura comercial & & & \\
\hline & & & & & 4. Nivel de acceso a capitales privados & & & \\
\hline \multirow{4}{*}{ 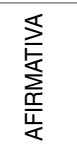 } & \multirow{4}{*}{$\begin{array}{l}16 \text { países menos adelantados, con } \\
\text { representatividad principalmente de África } \\
\text { Subsahariana }\end{array}$} & & & & 1. Nivel de endeudamiento externo & & & \\
\hline & & & & & 2. Nivel de apertura comercial & & & \\
\hline & & & & & 3. Nivel de acceso a capitales privados & & & \\
\hline & & & & & 4. Volumen del PIB per cápita & & & \\
\hline \multirow{4}{*}{ 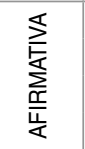 } & \multirow{4}{*}{$\begin{array}{l}19 \text { países pequeños, Estados insulares en } \\
\text { desarrollo, con representatividad principalmente } \\
\text { de América Latina y Caribe y Asia Oriental y } \\
\text { Pacífico. }\end{array}$} & & & & 1. Nivel de endeudamiento externo & & & \\
\hline & & & & & 2. Volumen del PIB per cápita & & & \\
\hline & & & & & 3. Nivel de apertura comercial & & & \\
\hline & & & & & 4. Nivel de acceso a capitales privados & & & \\
\hline \multirow{4}{*}{ 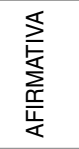 } & \multirow{4}{*}{$\begin{array}{l}16 \text { países en desarrollo sin litoral con } \\
\text { representatividad principalmente de África } \\
\text { Subsahariana, Europa y Asia Central }\end{array}$} & & & & 1. Nivel de apertura comercial & & & \\
\hline & & & $A P$ & & 2. Nivel del PIB per cápita & & & \\
\hline & & & APLIC & & 3. Nivel de endeudamiento externo & & & \\
\hline & & & & & 4. Nivel de acceso a capitales privados & & & \\
\hline$\$$ & & & & & 1. Nivel de endeudamiento externo & & & \\
\hline 莚 & 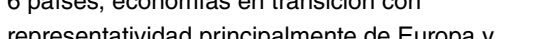 & & & & 2. Nivel de apertura comercial & & & \\
\hline$\underset{\cong}{\geqq}$ & Asia Central & & & & 3. Nivel del PIB per cápita & & & \\
\hline & & & & & 4. Nivel de acceso a capitales privados & & & \\
\hline$\$$ & & & & & 1. Nivel de endeudamiento externo & & & \\
\hline 妾 & $\begin{array}{l}12 \text { paises de Asia Oriental y Pacifico con } \\
\text { representatividad princinalmente de países en }\end{array}$ & & & & 2. Nivel del PIB per cápita & & & \\
\hline$\sum_{\underline{I}}$ & desarrollo & & & & 3. Nivel de apertura comercial & & & \\
\hline 峞 & & & & & 4. Nivel de acceso a capitales privados & & & \\
\hline$\leqslant$ & 13 países de Europa y Asía Central, con principal & & & & 1. Nivel de apertura comercial & & & \\
\hline 交 & representatividad de países en desarrollo, & & & & 2. Nivel de endeudamiento externo & & & \\
\hline$\sum_{\underline{I}}^{=}$ & economías de transición y países en desarrollo & & & & 3. Nivel de acceso a capitales privados & & & \\
\hline 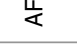 & sin litoral. & & & & 4. Nivel del PIB per cápita & & & \\
\hline$\$$ & & & & & 1. Nivel del PIB per cápita & & & \\
\hline 交 & 19 paises de America Lailna y caride con & & & & 2. Nivel de acceso a capitales privados & & & \\
\hline$\sum_{\underline{I}}$ & pegueños Fstados insulares & & & & 3. Nivel de apertura comercial & & & \\
\hline 蓆 & & & & & 4. Nivel de endeudamiento externo & & & \\
\hline$\$$ & & & & & 1. Nivel de endeudamiento externo & & & \\
\hline 安 & 6 paises de Oriente Medio y Norte de Africa con & & & & 2. Nivel de apertura comercial & & & \\
\hline 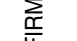 & representatividad principaimente de paises en & & & & 3. Nivel de acceso a capitales privados & & & \\
\hline 崖 & & & & & 4. Nivel del PIB per cápita & & & \\
\hline$\$$ & & & & & 1. Nivel de endeudamiento externo & & & \\
\hline 安 & 6 países de Asia Meridional con representatividad & & & & 2. Nivel de apertura comercial & & & \\
\hline$\sum_{\cong}^{\cong}$ & principalmente de países en desarrollo. & & & & 3. Nivel del PIB per cápita & & & \\
\hline 岩 & & & & & 4. Nivel de acceso a capitales privados & & & \\
\hline & & & & & 1. Nivel de apertura comercial & & & \\
\hline 交 & 20 paises de Atrica Subsahariana con & & & & 2. Nivel de endeudamiento externo & & & \\
\hline 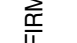 & $\begin{array}{l}\text { excepto economías en transición. } \\
\text { entreses, }\end{array}$ & & & & 3. Nivel de acceso a capitales privados & & & \\
\hline 宸 & & & & & 4. Nivel del PIB per cápita & & & \\
\hline
\end{tabular}


representatividad principalmente de África Subsahariana, el nivel de ajuste del modelo es bajo. El resultado confirma la hipótesis nuevamente, y en comparación con la submuestra global el mayor volumen de endeudamiento refuerza su correlación con las entradas de Ayuda Oficial al Desarrollo, y tanto la apertura comercial, el menor acceso a capitales privados y el PIB per cápita se reducen.

El grado de influencia y significación dentro del modelo muestra, en todos los años y en el nivel de renta baja, que la asignación de AOD se ve influenciada principalmente por el volumen de endeudamiento exterior, la menor apertura comercial, el acceso a capitales privados y el crecimiento del PIB. En este sentido se observa el impacto de los planes de condonación de deuda llevados a cabo durante el periodo (Bangladés 2002-2004, Nigeria, Zambia, Etiopía, Ghana, Siria 2005-2008, Argentina 2005-2010, Honduras, Haití, México, Perú y Congo 2010).

La asignación de la AOD en los países menos adelantados ha supuesto en torno a un tercio del total durante el periodo, con el mayor desembolso de AOD per cápita y con una gran concentración, con cerca del 50 por 100 en siete países (Afganistán, Etiopía, Congo, Tanzania, Bangladés, Mozambique y Uganda); en torno a un 75 por 100 subvencionada con un importante grado de concentración de donantes, Estados Unidos, con Reino Unido en bilateral, y Asociación Internacional de Fomento, con Comunidad Europea en multilateral. El principal destino de la Ayuda Oficial al Desarrollo ha sido a proyectos de infraestructuras sociales en torno al 45 por 100 (en educación, salud, gobierno y acceso a agua), seguidos de ayuda económica (17 por 100), ayuda humanitaria (10 por 100 ), ayuda productiva (9 por 100), condonación de deuda (4 por 100) y resto (15 por 100).
En el análisis de la muestra de países pobres muy endeudados de 15 países, con representatividad principalmente de África Subsahariana, el nivel de ajuste del modelo es bajo. El resultado confirma la hipótesis nuevamente, y si bien comparte el 56 por 100 del total de países con la submuestra de países menos adelantados, el resultado varía ligeramente por el peso del resto de países. En comparación con la submuestra global, el mayor volumen de endeudamiento refuerza su correlación con las entradas de AOD, y tanto la apertura comercial como el menor acceso a capitales privados y el PIB per cápita se reducen.

Con la inclusión de los países de la región de América Latina y Caribe se incrementa el peso del endeudamiento; al igual que el grado de influencia y significación dentro del modelo muestra en todos los años, aunque no en el nivel de renta, que la asignación de AOD se ve atraída principalmente por el mayor volumen de deuda externa, el menor PIB per cápita, la menor apertura comercial y el menor acceso a capitales privados, tal como se observa en el Cuadro 1.

Por otro lado, en el análisis de la muestra de pequeños Estados insulares en desarrollo de 19 países, con representatividad principalmente de América Latina y Caribe, África Subsahariana, Asia Oriental y Pacífico, el nivel de ajuste del modelo es medio. Las mayores correlaciones se dan con respecto al menor PIB per cápita y al menor acceso a capitales privados, y el resto se reducen (Anexo 3).

La significación por nivel de renta es baja en el modelo. El grado de influencia y significación dentro del modelo muestra que la asignación de AOD se ve influenciada principalmente por el mayor endeudamiento exterior, el menor PIB per cápita, la menor apertura comercial y, finalmente, el menor acceso a los capitales $\triangleright$ 
privados. Tal como se observa en el Cuadro 1, estos países han mostrado un crecimiento importante en las entradas de AOD, motivadas por la Ayuda Multilateral a Filipinas, las condonaciones de deuda a países de América Latina y Caribe y África Subsahariana, así como el creciente incremento de AOD hacia países menos adelantados, principalmente situados en África Subsahariana.

Respecto al análisis de la muestra de países en desarrollo sin litoral de 16 países, con representatividad principalmente de Europa, Asia Central y África Subsahariana, el nivel de ajuste del modelo es bajo. Igualmente se confirma la hipótesis nuevamente, tal y como muestran las correlaciones de la menor apertura comercial, el menor PIB per cápita, el mayor volumen de endeudamiento y el menor acceso a capitales privados. Esto se cumple de manera coherente por años excepto en 2005 , pero no por los diferentes niveles de renta.

En el análisis de la muestra de economías en transición de 6 países, con representatividad principalmente de Europa y Asia Central, el nivel de ajuste del modelo es bajo. El grado de influencia y significación dentro del modelo muestra en el nivel de renta, aunque no por años, que la asignación de AOD se ve influenciada principalmente por el mayor volumen de endeudamiento, la menor apertura comercial, el menor PIB per cápita y el menor acceso a capitales privados (Cuadro 1).

En el análisis de la muestra de Asia Oriental y Pacífico de 12 países, con representatividad principalmente de países en desarrollo, el nivel de ajuste del modelo es bajo. Las correlaciones evidencian que la AOD puede estar influenciada por el volumen de endeudamiento, el menor PIB per cápita, la menor apertura comercial y el menor acceso a capitales privados (Cuadro 1). Esto se cumple de manera coherente por niveles de renta, pero no por años. En la región, el mayor empuje se ha llevado a cabo por el crecimiento de la asistencia humanitaria y la ayuda multilateral, principalmente en Indonesia, Vietnam, Filipinas y Corea.

En el análisis de la muestra de Europa y Asia Central de 13 países, con representatividad de países en desarrollo, economías en transición y países en desarrollo sin litoral principalmente y con el nivel de ajuste del modelo bajo, las correlaciones del modelo señalan que la AOD puede estar influenciada por la menor apertura comercial, el mayor volumen de endeudamiento externo y el menor acceso a capital privado, principalmente. Esto se cumple de manera coherente por años, salvo en 2000 y 2005, y en el nivel de renta baja. Durante el periodo se produjo una reducción de las entradas de AOD, por la reducción de la ayuda bilateral de la UE a proyectos en la región y por la incorporación de varias economías a la UE en 2004 y a la OCDE, a pesar de las condonaciones de deuda a la Federación Rusa y a Serbia durante el periodo.

En el análisis de la muestra de América Latina y Caribe de 29 países, con representatividad de países en desarrollo y pequeños Estados insulares en desarrollo, principalmente, y con el nivel de ajuste del modelo medio, el resultado confirma la hipótesis nuevamente, tal y como muestran las correlaciones con las entradas de AOD del menor PIB per cápita, el menor acceso a capital privado y de manera similar la menor apertura comercial y el mayor volumen de endeudamiento externo. Esto se cumple de manera homogénea por años y diferentes niveles de renta. La región ha sufrido una reducción de entradas hasta 2005 y una recuperación posterior debido a la ayuda multilateral regional, con el crecimiento de la asistencia humanitaria a Chile en 2010 , las $\triangleright$ 
condonaciones de deuda a Argentina en 2005 y 2010 y a Honduras, Haití, México y Perú.

En el análisis de la muestra de Oriente Medio y Norte de África de 6 países, con representatividad principalmente de países en desarrollo, el nivel de ajuste del modelo es bajo. De acuerdo con las correlaciones, se evidencia que la AOD está influenciada por el mayor volumen de endeudamiento externo, la menor apertura comercial y el menor acceso a capital privado, principalmente (Cuadro 1). Esto se cumple de manera homogénea por niveles de renta, pero no por años. En dicha región se ha recuperado el volumen de entradas de AOD por el crecimiento de la ayuda bilateral para proyectos financiada principalmente por la Liga Árabe y por países con intereses poscoloniales, y también, en menor medida, por la asistencia humanitaria a Egipto, Siria y Marruecos en 2000 y la condonación de deuda a Siria en 2005.

En el análisis de la muestra de Asia Meridional de 6 países, con representatividad de países en desarrollo principalmente, el nivel de ajuste del modelo es muy bajo. El resultado de las correlaciones con las entradas de AOD de todas las variables evidencian la influencia principalmente del mayor volumen de endeudamiento, la menor apertura comercial, el menor PIB per cápita y el menor acceso a capitales privados (Cuadro 1).

La región ha tenido un ligero crecimiento por la ayuda bilateral a proyectos con origen en EEUU para Afganistán tras la guerra y también a Pakistán. El crecimiento de la ayuda multilateral ha sido hacia India principalmente, también a Pakistán y Afganistán en menor medida, con planes de condonación de deuda de Bangladés en 2002-2004 y Pakistán en 2003-2008.

Por último, en el análisis de la muestra de África Subsahariana de 20 países, con representatividad de todos los tipos de países salvo economías en transición y con el nivel de ajuste del modelo medio, el resultado confirma la hipótesis nuevamente, como muestran las correlaciones con las entradas de AOD con todas las variables.

Se ha producido un gran crecimiento en la región motivado por el aumento de la AOD debido al cambio de su política de asignación hacia los países menos adelantados; además de los crecimientos por condonaciones de deuda a países como Nigeria, Zambia, Etiopía y Ghana en 2005-2008 y Congo en 2010. También han sido impulsados por el Plan de África 2009 a 2012, lo cual en su conjunto ha supuesto que se dupliquen los flujos de entrada de Ayuda Oficial al Desarrollo.

\section{Reflexiones finales}

El objetivo principal del estudio es el análisis de la influencia que sobre los flujos de entradas de AOD en la economía han mostrado una serie de variables independientes representativas de los principales factores descriptivos de la misma. Por ello se recuerda que la hipótesis nula indica que la asignación de AOD se tiene con un carácter compensatorio, siendo distribuida allí donde existe menor acceso a financiación privada e internacional, tomando para ello una muestra global tanto a nivel regional como por tipo de países y niveles de renta.

La falta de fuentes de datos con el suficiente detalle ha limitado el análisis de variables a nivel general, impidiendo el detalle de tipología de AOD, de sus fuentes, de sus donantes y por tipo de actividad. Si bien, como ya se comentó, resulta limitada la exactitud y el detalle que se puede extraer tanto de los criterios de distribución de los flujos de entrada de AOD realizadas por los donantes como de las estrategias de $D$ 
desarrollo adoptadas por los países receptores y de la eficacia sobre las mismas.

A nivel global, el resultado obtenido tanto en el modelo inicial como en el de submuestras corrobora la experiencia empírica y pone de manifiesto la existencia de una serie de factores con un nivel de influencia representativo y significativo sobre el volumen de entradas de AOD para el conjunto de países y durante el periodo de estudio.

\section{Bibliografía}

[1] AABY, N. E. y SLATER, S.F. (1989). «Management Influences on Export Performance: A Review of the Empirical Literature 1978-1988». International Marketing Review, vol. 6, n. 94.

[2] ALONSO, J. A. y DONOSO, V. (1994). Competitividad de Empresa Exportadora Española. Madrid: Instituto Español de Comercio Exterior.

[3] ARTARAZ, M. (2002). «Teoría de las tres dimensiones del desarrollo sostenible». Revista Ecosistemas, vol. 11, n. .2 .

[4] AZARIADIS, C. (2006). «The Theory of poverty traps. What have we learned». Poverty traps. Priceton University Press.

[5] BANCO IBERAMERICANO DE DESARROLLO (2007). Triennial Central Bank Survey of Foreign Exchange and Derivatives Market Activity. Disponible en: http://www.iadb.org (Recuperado: 2014, 5 de enero).

[6] BANCO MUNDIAL (2011). Informe de Desarrollo Mundial. El estado en un mundo en transformación.

[7] BANCO MUNDIAL (2011). Datos sobre Migración y Remesas 2011, 2. edición, Unidad de Migración y Remesas del Banco Mundial.

[8] BANCO MUNDIAL (2014). Reseña sobre Migración y Desarrollo 2014. Comunicado de prensa.

[9] BANDYOPADHYAY, B. y WALL, J. (2007). «The Determinants of Aid in the Post-Cold War Era». Federal Reserve Bank of St. Louis.
Review, November/December, vol. 89, n.. 6 , pp. 533-547.

[10] BEVAN, A. A. y ESTRIN, S. (2000). Patterns of foreign direct investment and trade in central and eastern europe, mimeo.

[11] BORJAS, G. J. (1989). «Economic theory and international migration». International migration review.

[12] BOWLES, S.; DURLANF, S. N. y HOFF, K. (2006). Poverty traps. Princeton University Press.

[13] BUCKLEY, P. J. y CASSON, M. (1976). The future of the multinational Enterprise. Ed. MacMillan.

[14] CALDUCH CERVERA, R. (1991). Relaciones Internacionales. Ed. Ediciones Ciencias Sociales.

[15] CASTELAZO, J. R. (2007). Administración Pública, una visión de Estado. INAP, México.

[16] CAVUSGIL, T. y ZOU, S. (1994). "Marketing Strategy-Performance Relationship: An Investigation of the Empirical Link in Export Market Ventures». Journal of Marketing n. -58 , pp. 1-21.

[17] COHEN, G. A. (2001). If you are Egalitarian, How come you are so rich? Harvard University Press. New Ed.

[18] DIAZ MIER, M. A. y CAVIEDES, A. (1996). La OMC y sus Conferencias Ministeriales. Trabajo de Investigación Universidad de Alcalá de Henares.

[19] DORNBUSCH et al. (1983). «The Black Market for Dollars in Brazil». National Burou of Economic Research, n. 590.

[20] DUNNING, J. (1973). "The determinants of International production». Ed. Oxford Economic papers, n. 25 . Oxford University.

[21] DURÁN HERRERA, J. J. (1994). «Internacionalización de la empresa española en la crisis. Exportaciones e inversión directa». Revista Economistas.

[22] EATON WILLIAMSON, O. (2000). «The new institutional economics: Taking stock, Looking Ahead». Journal of Economic Literature, vol. 38, pp. 595-613.

[23] FAINI, R. y VENTURINI, A. (1994). «Migration and growth: the experience of Southern Europe». Discussion Paper, n. 964. London: Centre for Economic Policy Research.

[24] FONDO MONETARIO INTERNACIONAL (2014). Global Financial Stability Report: $\square$ 
Moving from liquidity to growth-driven markets. Washington, EEUU. Disponible en: $h$ ttp:// www.fmi.org. (Recuperado: 2014, el 5 de enero).

[25] GARCIA ARIAS, J. (2008). «Finanzas Internacionales y Desarrollo. ¿Oportunidad o Amenaza?». CEPAL n.o 96, p. 161.

[26] GASTANAGA, V. M.; NUGENT, J. B. y PASHAMOVA, B. (1998). «Host Country Reforms and FDI Inflows: How Much Difference Do They Make?». World Development, vol. 26, n. -7 , pp. 1299-1314.

[27] GREENHILL, R. y ALI, A. (2013). Paying for progress: how will emerging post-2015 goals be financed in the new aid landscape? Londres: Overseas Development Institute.

[28] HARROD, R. F. (1939). «An Essay in Dynamic Theory». The Economic Journal, vol.49, n.-0193, pp 14-33.

[29] HOLLAND, D. y PAIN, N. (1998). The determinants and impact of foreign direct investment in the transition economies: a panel data analysis. National Institute of Economic and Social Research.

[30] HYMER, S. H. (1976). The international Operations of National firms: $A$ study of Foreign Investment. Massachusetts Institute of Technology.

[31] JOHANSON, J. y MATTSON, L. G. (1988). Internationalization in Industrial Systems. A Network Approach. London: Academic Press.

[32] KAHAI, S. K. (2011). «Traditional And NonTraditional Determinants Of Foreign Direct Investment In Developing Countries». Journal Of Applied Business Research, vol. 20, n. -1 .

[33] KATSELI, L. y GLYTSOS, N. (1986). «Theoretical and Empirical Determinants of International Labour Mobility: A GreekGerman Perspective». Working Paper, n. .148. Centre for Economic Policy Research.

[34] KOCH, D. J.; DREHER, A.; NUNNENKAMP, P. y THIELE, R. (2008). «Keeping a Low Profile: What Determines the Allocation of Aid by NonGovernmental Organizations?». Kiel Institute Working Paper, n. 1406.

[35] KUCERA, D. (2001). «The effects of core workers rights on labour costs and foreign direct investment: Evaluating the conventional wisdom». IILS. Discussion paper, DP.130. 2001.
[36] LANDRY, A. (1909). «Les trois théories de la population». Revue Scientia, vol. 3, n.ㅇ 6, p. 121. Francia.

[37] LUCAS, R. E. (1988). "On the mechanics of Economic development». Journal of Monetary Economics, vol. 22, n. 1 , pp. 3-42.

[38] MARCUELLO SERVOS, C.; CLEMENTE LOPEZ, J. Y FILGUEIRA CHIOSSONI, M. (2010). Dos caras de una misma moneda: Ayuda Oficial para el Desarrollo y Deuda Externa en la cooperación para el desarrollo. Universidad de Zaragoza.

[39] MARTÍNEZ CARRERAS, J. U. (1992). Historia del Colonialismo y la descolonización. Madrid: Ed. Complutense S.A.

[40] MASSEY, D.; ARANGO, J. et al. (1998). Worlds in motion. Understanding international migration at the end of the millenium. Oxford: Clarendon Press.

[41] MOGROVEJO, J. A. (2005). «Factores determinantes de la inversión extranjera directa en algunos países de latinoamérica». Estudios Económicos de Desarrollo Internacional. $A E E A D E$, vol. 5-2.

[42] NACIONES UNIDAS (2002). Conferencia Internacional de Financiación para el Desarrollo. La Conferencia de Monterrey. Disponible en: http://www.un.org/es/conf/ ffd/2002/

[43] NACIONES UNIDAS (2005). Declaración de París.

[44] NACIONES UNIDAS (2008). Programa de Acción de Accra.

[45] NACIONES UNIDAS (2012). Realizing the future we want for all, UN System task team on the Post2015 UN Development Agenda. June 2012. Disponible en: www.un.org (Recuperado: 2014, el 5 de enero).

[46] NONNENBERG, M. y MENDONCA, M. (2004). «The determinants of direct foreign investment in developing countries». January. Working Paper. Institute of Applied Economic Research.

[47] NUNES, C. L., OSCATEGUI, J. y PESCHIERA, J. (2006). Determinants of FDI in Latin America. Documento de trabajo 252.

[48] OCDE (2011). Foro de Alto Nivel de Busan. Disponible en: http://www.oecd.org/dac/ effectiveness/34580968.pdf (Recuperado: 2014, el 5 de enero). 
[49] OMC (2014). Informe sobre el Comercio Mundial. Disponible en: $h$ ttp://www.wto.org (Recuperado: 2014, el 5 de enero).

[50] PÉREZ DÍAZ, J. y MACINNES, J. (2009). La tercera revolución de la modernidad; la revolución reproductiva. Centro de Investigaciones Sociológicas.

[51] POELHEKKE, S. y VAN DER PLOEG, F. (2008). «Growth, Foreign Direct Investment and Urban Concentrations: Unbundling Spatial Lags«. Working Paper n.o 195. De Nederlandsche Bank NV.

[52] PORTER, R. C. (1973). A model of labor migration and urban unemployment in less developed countries. Center for Research on Economic Development. DP- 29.

[53] PREBISH, R. (1950). The economic development of Latin America and its Principal Problems. United Nations, New York.

[54] RED EUROSUR (2014). Financiación Internacional para programas mundiales prioritarios. El papel de la Ayuda Oficial al Desarrollo. Disponible en: http://www.eurosur.org/futuro/ fut78.htm (Recuperado: 2014, el 10 de diciembre).

[55] RIALP, A. y RIALP, J. (2001). Conceptual frameworks on SMEs' internationalization: Past, present and future trends of research. Emerald Group Publishing Limited.

[56] RODRIK, D. (2012). La paradoja de la globalización. Antoni Bosh Editor.

[57] ROMER, P. (1986). «Increasing returns and long-run growth». Journal of Policital Economy, vol. 94 , n. 5 , pp. $1002-1037$.

[58] ROSENSTEIN-RODAN (1943). «The problems of industrialization of Eastern and South-Eastern Europe». The Economic Journal, $\mathrm{n} .{ }^{\circ} 53$.

[59] SASKIA, C.; VAN DER ZEE, S. C.; HOEK, G.; HARRSSEMA, H. y BRUNEKREEF B. (1998). Characterization of particulate air pollution in urban and non-urban areas in the Netherlands. Atmos Environ.

[60] SEN, A. (2000). El desarrollo como libertad. Madrid: Ed. Planeta.

[61] SHARMA, K.; KASHYAP, A.; MONTES, M. F. y LADD, P. (2001). Realizing themdevelopment
Potential of Diasporas. United Nations University Press.

[62] SOLOW (1956). «A contribution to the Theory of Economic Growth». Quarterly Journal of Economics.

[63] STARK, O. (1993). La migración y el trabajo. Madrid: MTAS.

[64] STEIN, P.; GOLAND, T. y SCHIFF, R. (2010). Two trillion and counting: assessing the credit gap for micro, small, and medium-sized enterprises in the developing world. Washington D.C: Corporación Financiera Internacional y McKinsey \& Company.

[65] STEWART, F. y STREETEN, P. (1976). «New strategies for Development: Poverty, Income Distribution, and growth». Oxford Economic Papers, vol. 28, n.ㅇ․ 3 .

[66] STIGLITZ, J. E. (1999). «El papel del Gobierno en el Desarrollo Económico». Cuadernos de Economía, vol. 18, n. 30 . Bogotá.

[67] VAN DE KAA, D. J. (2002). The Idea of a Second Demographic Transition in Industrialized Countries. Paper presented at the Sixth Welfare Policy Seminar of the National Institute of Population and Social Security, Tokio, Japón, 29 de enero de 2002.

[68] VERNON, R. (1979). «The product life-cycle in a new international environment». Oxford Bulletin of Economics and Statistics, n. -41.

[69] WILLIAMSON, J. (1993). «Democracy and the Washington Consensus». World Development, n. - 21. Londres.

[70] WILLIAMSON, O. E. (1974). «The economics of antitrust: Transaction cost considerations». University of Pensilvania, Law review, vol. 122, n.으 6, pp.1439-1496.

[71] YANG, D. (2006). «International Migration, Remittances and Household Investment: Evidence from Philippine Migrants' Exchange Rate Shocks». The Economic Journal Royal Economic Society, vol. 118, n.․ 528, pp. 591-630.

[72] YANGUAS, L. y CAVIEDES, A. (2017). «Impacto de las inversiones privadas en el desarrollo de los países en desarrollo». Boletín Económico: Información comercial española, n. .03093 . 


\section{ANEXO I \\ DEFINICIÓN DE LAS VARIABLES}

\begin{tabular}{|c|c|c|}
\hline Variable & Descripción & Fuente \\
\hline AOD & $\begin{array}{l}\text { Net official } \\
\text { development } \\
\text { assistance } \\
\text { and official aid } \\
\text { received } \\
\text { (current US\$) }\end{array}$ & $\begin{array}{l}\text { Net official development assistance is disbursement flows (net of repayment of principal) that meet the } \\
\text { DAC definition of ODA and are made to countries and territories on the DAC list of aid recipients. Net } \\
\text { official aid refers to aid flows (net of repayments) from official donors to countries and territories in } \\
\text { part II of the DAC list of recipients: more advanced countries of Central and Eastern Europe, the countries } \\
\text { of the former Soviet Union, and certain advanced developing countries and territories. Official aid is } \\
\text { provided under terms and conditions similar to those for ODA. Part II of the DAC List was abolished } \\
\text { in } 2005 \text {. The collection of data on official aid and other resource flows to Part II countries ended with } \\
2004 \text { data. Data are in current U.S. dollars. }\end{array}$ \\
\hline PIBpc & $\begin{array}{l}\text { GDP per } \\
\text { capita (current } \\
\text { US\$) }\end{array}$ & $\begin{array}{l}\text { GDP per capita is gross domestic product divided by midyear population. GDP is the sum of gross } \\
\text { value added by all resident producers in the economy plus any product taxes and minus any subsidies } \\
\text { not included in the value of the products. It is calculated without making deductions for depreciation of } \\
\text { fabricated assets or for depletion and degradation of natural resources. Data are in current U.S. } \\
\text { dollars. }\end{array}$ \\
\hline EXP & $\begin{array}{l}\text { Exports of } \\
\text { goods and } \\
\text { services } \\
(\% \text { of GDP) }\end{array}$ & $\begin{array}{l}\text { Exports of goods and services represent the value of all goods and other market services provided } \\
\text { to the rest of the world. They include the value of merchandise, freight, insurance, transport, travel, } \\
\text { royalties, license fees, and other services, such as communication, construction, financial, information, } \\
\text { business, personal, and government services. They exclude compensation of employees and } \\
\text { investment income (formerly called factor services) and transfer payments. }\end{array}$ \\
\hline KPRIV & $\begin{array}{l}\text { Private capital } \\
\text { flows, total } \\
(\% \text { of GDP) }\end{array}$ & $\begin{array}{l}\text { Private capital flows consist of net foreign direct investment and portfolio investment. Foreign direct } \\
\text { investment is net inflows of investment to acquire a lasting management interest (10 percent or more } \\
\text { of voting stock) in an enterprise operating in an economy other than that of the investor. It is the sum } \\
\text { of equity capital, reinvestment of earnings, other long-term capital, and short-term capital as shown in } \\
\text { the balance of payments. The FDI included here is total net, that is, net FDI in the reporting economy } \\
\text { from foreign sources less net FDI by the reporting economy to the rest of the world. Portfolio } \\
\text { investment excludes liabilities constituting foreign authorities' reserves and covers transactions } \\
\text { in equity securities and debt securities. }\end{array}$ \\
\hline DEXT & $\begin{array}{l}\text { Net flows on } \\
\text { external debt, } \\
\text { total (NFL, } \\
\text { current US\$) }\end{array}$ & $\begin{array}{l}\text { Net flows on external debt are disbursements on long-term external debt and IMF purchases minus } \\
\text { principal repayments on long-term external debt and IMF repurchases up to } 1984 \text {. Beginning in } 1985 \\
\text { this line includes the change in stock of short-term debt (including interest arrears for long-term debt). } \\
\text { Thus, if the change in stock is positive, a disbursement is assumed to have taken place; if negative, a } \\
\text { repayment is assumed to have taken place. Long-term external debt is defined as debt that has an } \\
\text { original or extended maturity of more than one year and that is owed to nonresidents by residents of } \\
\text { an economy and repayable in foreign currency, goods, or services. Data are in current U.S. dollars. }\end{array}$ \\
\hline POB & $\begin{array}{l}\text { Population, } \\
\text { total }\end{array}$ & $\begin{array}{l}\text { Total population is based on the de facto definition of population, which counts all residents regardless } \\
\text { of legal status or citizenship, except for refugees not permanently settled in the country of asylum, } \\
\text { who are generally considered part of the population of their country of origin. The values shown are } \\
\text { midyear estimates. }\end{array}$ \\
\hline
\end{tabular}




\section{UN MODELO PARA EL ESTUDIO DE LAS VARIABLES ECONÓMICAS QUE INFLUYEN...}

\section{ANEXO II \\ PAÍSES POR MUESTRAS}

\begin{tabular}{|c|c|c|}
\hline Muestra & Número & Países \\
\hline Global & 86 & $\begin{array}{l}\text { Cambodia, China, Fiji, Indonesia, Lao PDR, Malaysia, Mongolia, Papua New Guinea, } \\
\text { Philippines, Solomon Islands, Thailand, Vietnam, Albania, Armenia, Azerbaijan, Belarus, } \\
\text { Bosnia and Herzegovina, Croatia, Georgia, Kazakhstan, Kyrgyz Republic, Macedonia, } \\
\text { Moldova, Turkey, Ukraine, Egypt, Arab Rep., Jordan, Morocco, Syrian Arab Republic, } \\
\text { Tunisia, Yemen, Rep., Antigua and Barbuda, Argentina, Barbados, Belize, Bolivia, Brazil, } \\
\text { Chile, Colombia, Costa Rica, Dominica, Dominican Republic, Ecuador, El Salvador, } \\
\text { Grenada, Guatemala, Guyana, Haiti, Honduras, Jamaica, Mexico, Nicaragua, Panama, } \\
\text { Paraguay, Peru, St. Kitts and Nevis, St. Lucia, St. Vincent and the Grenadines, Uruguay, } \\
\text { Venezuela, RB, Bangladesh, Bosnia and Herzegovina, India, Maldives, Pakistan, Sri } \\
\text { Lanka, Angola, Botswana, Cameroon, Cape Verde, Ghana, Guinea, Kenya, Lesotho, } \\
\text { Mauritius, Mozambique, Nigeria, Rwanda, Seychelles, Sierra Leone, South Africa, } \\
\text { Sudan, Swaziland, Tanzania, Uganda, Zambia. }\end{array}$ \\
\hline TE - Transition economies & 5 & Albania, Belarus, Bosnia and Herzegovina, Croatia, Ukraine. \\
\hline DC - Developing countries & 85 & $\begin{array}{l}\text { Cambodia, China, Fiji, Indonesia, Lao PDR, Malaysia, Mongolia, Papua New Guinea, } \\
\text { Philippines, Solomon Islands, Thailand, Vietnam, Albania, Armenia, Azerbaijan, Belarus, } \\
\text { Bosnia and Herzegovina, Croatia, Georgia, Kazakhstan, Kyrgyz Republic, Macedonia, } \\
\text { Moldova, Turkey, Ukraine, Egypt, Arab Rep., Jordan, Morocco, Syrian Arab Republic, } \\
\text { Tunisia, Yemen, Rep., Antigua and Barbuda, Argentina, Barbados, Belize, Bolivia, Brazil, } \\
\text { Colombia, Costa Rica, Dominica, Dominican Republic, Ecuador, El Salvador, Grenada, } \\
\text { Guatemala, Guyana, Haiti, Honduras, Jamaica, Mexico, Nicaragua, Panama, Paraguay, } \\
\text { Peru, St. Kitts and Nevis, St. Lucia, St. Vincent and the Grenadines, Uruguay, } \\
\text { Venezuela, Bangladesh, Bosnia and Herzegovina, India, Maldives, Pakistan, Sri Lanka, } \\
\text { Angola, Botswana, Cameroon, Cape Verde, Ghana, Guinea, Kenya, Lesotho, Mauritius, } \\
\text { Mozambique, Nigeria, Rwanda, Seychelles, Sierra Leone, South Africa, Sudan, } \\
\text { Swaziland, Tanzania, Uganda, Zambia. }\end{array}$ \\
\hline $\begin{array}{l}\text { HIPC-Highly indebted poor } \\
\text { countries }\end{array}$ & 15 & $\begin{array}{l}\text { Bolivia, Guyana, Haiti, Honduras, Nicaragua, Cameroon, Ghana, Guinea, Lesotho, } \\
\text { Mozambique, Rwanda, Sierra Leone, Sudan, Tanzania, Uganda, Zambia. }\end{array}$ \\
\hline $\begin{array}{l}\text { LDC - Least developed } \\
\text { countries }\end{array}$ & 16 & $\begin{array}{l}\text { Cambodia, Lao PDR, Solomon Islands, Haiti, Yemen, Rep., Bangladesh, Angola, } \\
\text { Guinea, Lesotho, Mozambique, Rwanda, Sierra Leone, Sudan, Tanzania, Uganda, } \\
\text { Zambia. }\end{array}$ \\
\hline $\begin{array}{l}\text { LDS - Landlocked } \\
\text { developing states }\end{array}$ & 16 & $\begin{array}{l}\text { Lao PDR, Mongolia, Armenia, Azerbaijan, Kazakhstan, Kyrgyz Republic, Macedonia, } \\
\text { Moldova, Bolivia, Paraguay, Botswana, Lesotho, Rwanda, Swaziland, Uganda, Zambia. }\end{array}$ \\
\hline $\begin{array}{l}\text { SIDS - Small island } \\
\text { developing states }\end{array}$ & 19 & $\begin{array}{l}\text { Fiji, Papua New Guinea, Solomon Islands, Antigua and Barbuda, Barbados, Belize, } \\
\text { Dominica, Dominican Republic, Grenada, Guyana, Haiti, Jamaica, St. Kitts and Nevis, } \\
\text { St. Lucia, St. Vincent and the Grenadines, Maldives, Cape Verde, Mauritius, Seychelles. }\end{array}$ \\
\hline EAS - East Asia and Pacific & 12 & $\begin{array}{l}\text { Cambodia, China, Fiji, Indonesia, Lao PDR, Malaysia, Mongolia, Papua New Guinea, } \\
\text { Philippines, Solomon Islands, Thailand, Vietnam. }\end{array}$ \\
\hline $\begin{array}{l}\text { ECS - European } \\
\text { Cooperating States }\end{array}$ & 13 & $\begin{array}{l}\text { Albania, Armenia, Azerbaijan, Belarus, Bosnia and Herzegovina, Croatia, Georgia, } \\
\text { Kazakhstan, Kyrgyz Republic, Macedonia, Moldova, Turkey, Ukraine. }\end{array}$ \\
\hline $\begin{array}{l}\text { MEA - Middle East and } \\
\text { Africa }\end{array}$ & 6 & Egypt, Arab Rep., Jordan, Morocco, Syrian Arab Republic, Tunisia, Yemen, Rep. \\
\hline $\begin{array}{c}\text { LCN - Latin America and } \\
\text { Caribbean }\end{array}$ & 29 & $\begin{array}{l}\text { Antigua and Barbuda, Argentina, Barbados, Belize, Bolivia, Brazil, Chile, Colombia, } \\
\text { Costa Rica, Dominica, Dominican Republic, Ecuador, El Salvador, Grenada, Guatemala, } \\
\text { Guyana, Haiti, Honduras, Jamaica, Mexico, Nicaragua, Panama, Paraguay, Peru, } \\
\text { St. Kitts and Nevis, St. Lucia, St. Vincent and the Grenadines, Uruguay, Venezuela, RB. }\end{array}$ \\
\hline SAS - Sourthern Asia & 6 & Bangladesh, Bosnia and Herzegovina, India, Maldives, Pakistan, Sri Lanka. \\
\hline SSF - SouthSaharian Africa & 20 & $\begin{array}{l}\text { Angola, Botswana, Cameroon, Cape Verde, Ghana, Guinea, Kenya, Lesotho, Mauritius, } \\
\text { Mozambique, Nigeria, Rwanda, Seychelles, Sierra Leone, South Africa, Sudan, } \\
\text { Swaziland, Tanzania, Uganda, Zambia. }\end{array}$ \\
\hline
\end{tabular}


ANEXO III

RESULTADOS POR MUESTRAS

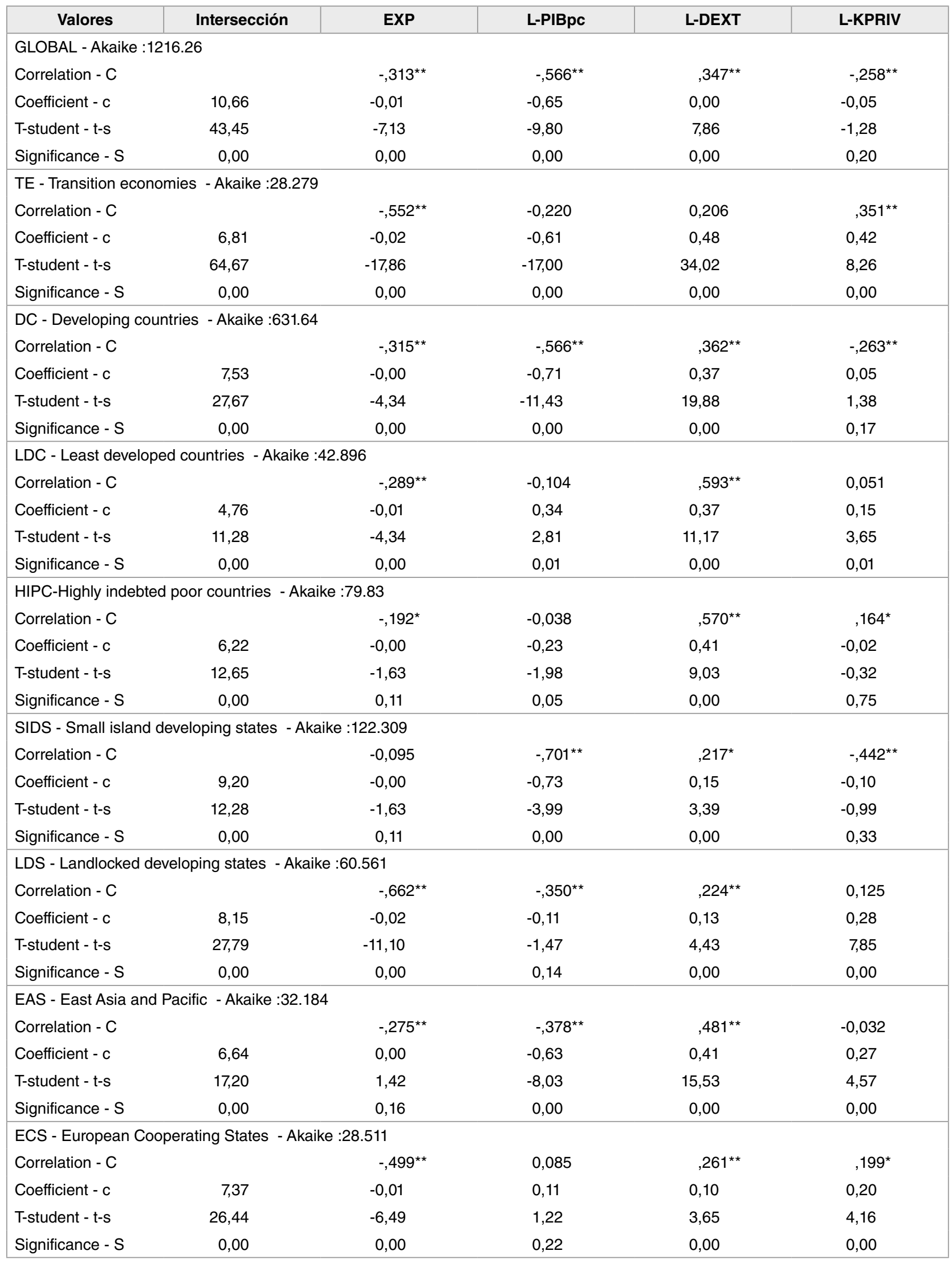


UN MODELO PARA EL ESTUDIO DE LAS VARIABLES ECONÓMICAS QUE INFLUYEN...

\begin{tabular}{|c|c|c|c|c|c|}
\hline Valores & Intersección & EXP & L-PIBpc & L-DEXT & L-KPRIV \\
\hline \multicolumn{6}{|c|}{ MEA - Middle East Africa - Akaike :27.33 } \\
\hline Correlation - C & & $-0,210$ & 0,175 &, $343^{*}$ & 0,227 \\
\hline Coefficient - c & 3,93 & 0,03 & 0,43 & 0,20 & 0,21 \\
\hline T-student - t-s & 11,19 & 22,41 & 7,42 & 10,18 & 8,21 \\
\hline Significance - S & 0,00 & 0,00 & 0,00 & 0,00 & 0,00 \\
\hline \multicolumn{6}{|c|}{ SAS - Sourthern Asia - Akaike :13.964 } \\
\hline Correlation - C & &,$- 885^{\star *}$ &,$- 783^{\star *}$ &, $781^{* *}$ &,$- 488^{* *}$ \\
\hline Coefficient - c & 4,70 & $-0,01$ & 0,19 & 0,36 & $-0,24$ \\
\hline T-student - t-s & 23,70 & $-16,41$ & 9,26 & 25,33 & $-5,89$ \\
\hline Significance - S & 0,00 & 0,00 & 0,00 & 0,00 & 0,00 \\
\hline \multicolumn{6}{|c|}{ SSF - SouthSaharian Africa - Akaike :140.743 } \\
\hline Correlation - C & &,$- 514^{\star *}$ &,$- 531^{\star *}$ &, $491^{* *}$ & $-0,102$ \\
\hline Coefficient - c & 6,09 & $-0,01$ & $-0,53$ & 0,51 & $-0,05$ \\
\hline T-student - t-s & 11,44 & $-3,21$ & $-5,10$ & 13,79 & $-0,83$ \\
\hline Significance - S & 0,00 & 0,00 & 0,00 & 0,00 & 0,41 \\
\hline \multicolumn{6}{|c|}{ LCN - Latin America and Caribbean - Akaike :100.905 } \\
\hline Correlation - C & &,$- 366^{\star \star}$ &,$- 575^{\star \star}$ &, $363^{* *}$ &,$- 417^{\star *}$ \\
\hline Coefficient - c & 9,10 & $-0,01$ & $-1,06$ & 0,33 & $-0,00$ \\
\hline T-student - t-s & 13,87 & $-4,76$ & $-6,63$ & 8,70 & $-0,03$ \\
\hline Significance - S & 0,00 & 0,00 & 0,00 & 0,00 & 0,97 \\
\hline
\end{tabular}

